\title{
Impact of body habitus on fluoroscopic radiation emission during minimally invasive spine surgery
}

\author{
Presented at the 2014 AANS/CNS Joint Section on Disorders of the Spine and Peripheral Nerves \\ Sunil Kukreja, MS, Justin Haydel, MD, Anil Nanda, MD, MPH, and Anthony H. Sin, MD \\ Department of Neurosurgery, Louisiana State University Health Science Center-Shreveport, Shreveport, Louisiana
}

\begin{abstract}
OBJECT Minimally invasive spine surgeries (MISSs) have gained immense popularity in the last few years. Concern about the radiation exposure has also been raised. The purpose of this study was to demonstrate the impact of body habitus on the radiation emission during various MISS procedures. The authors also aim to evaluate the effect the surgeon's experience has on the amount of radiation exposure during MISS especially with regard to patient size.
\end{abstract}

METHODS The authors conducted a retrospective analysis of 332 patients who underwent 387 MISS procedures performed at their institution from January 2010 to August 2013 by a single surgeon. The dose of radiation emission available from the fluoroscopic equipment was recorded from the electronic database. The authors analyzed mainly 3 procedure groups: microdiscectomy/decompression (MiDD, $n=211$ ) and transforaminal lumbar interbody fusion (TLIF) either with unilateral instrumentation (UnTLIF, $n=106$ ) or bilateral instrumentation (BiTLIF, $n=70$ ). The patients in each procedure group were divided into 6 categories based on the WHO criteria for obesity: underweight (body mass index $[\mathrm{BMI}]<18.50)$, normal (18.50-24.99), overweight (25.00-29.99), Class 1 obese (30.00-34.99), Class 2 obese (35.0039.99), and Class 3 obese (> 40.00).

RESULTS Patients who underwent BiTLIF had the highest median radiation exposure (113 mGy, SD 9.44), whereas microdiscectomy required minimal exposure (12.62 mGy, SD $2.75 \mathrm{mGy})$. There was a significant correlation between radiation emission and BMI of the patients during all MISS procedures $(p<0.05)$. The median radiation exposure was substantially greater with larger patients $(p \leq 0.001)$. In the analyses within the procedure groups, radiation exposure was found to be significantly high in patients who were severely obese (Class 2 and Class 3 obesity). The radiation emission was lower during the surgeries performed in 2013 than during those performed in 2010 especially in obese patients; however, this observation was not statistically significant.

CONCLUSIONS Body habitus of the patients has a substantial impact on radiation emission during MISS. Severe obesity $(\mathrm{BMI} \geq 35)$ is associated with a significantly greater risk of radiation exposure compared with other weight categories. Surgical experience seems to be associated with lower radiation emission especially in cases in which patients have a higher BMl; however, further studies should be performed to examine this effect.

http://thejns.org/doi/abs/10.3171/2014.10.SPINE14163

KEY WORDS minimally invasive spine surgery; radiation exposure; learning curve; obesity; body mass index; body habitus; technique

$\mathrm{M}$ INIMALLY invasive spine surgery (MISS) techniques have gained widespread popularity in recent years. Minimal soft-tissue dissection with a short hospital stay and short recovery period are the potential advantages of MISS. ${ }^{7}$ The advent of fluoroscopic guidance and endoscopic technology has played an important role in the evolution of these techniques over the course of the last several years. ${ }^{1,13}$ Simultaneously, there is also a growing concern of radiation exposure with MISS . ${ }^{3,6,12,14,15,23,24}$ Mariscalco et al ${ }^{12}$ compared the radiation exposure to surgeons during open and minimally invasive discectomy procedures, and they observed that the exposure was significantly higher for surgeons who performed surgeries with MISS techniques. However, dose

ABBREVIATIONS BiTLIF = bilateral TLIF; BMI = body mass index; $D D D=$ degenerative disc disease; MiDD = microdiscectomy/decompression; MISS = minimally invasive spine surgery; TLIF = transforaminal lumbar interbody fusion; UnTLIF = unilateral TLIF.

SUBMITTED February 10, 2014. ACCEPTED October 23, 2014.

INCLUDE WHEN CITING Published online December 5, 2014; DOI: 10.3171/2014.10.SPINE14163.

DISCLOSURE The authors report no conflict of interest concerning the materials or methods used in this study or the findings specified in this paper. 
and radiogenic risk have also been found within tolerable limits by some investigators. . $^{3,22}$

Spine surgeries in obese patients are technically more difficult, and the duration of the surgical procedure is also longer in these patients. ${ }^{5,20,28}$ Greater radiation exposure for obese patients has already been reported during various endoscopic or other minimally invasive procedures. ${ }^{8,25,29}$ Rampersaud et al. ${ }^{23}$ recorded greater radiation dose during open pedicle screw fixation in larger cadaveric specimens (> $150 \mathrm{lbs}$ ). Wang et al. ${ }^{28}$ reported a significantly higher fluoroscopic exposure time in overweight (body mass index [BMI] 25-30) and obese (BMI > 30) patients undergoing minimally invasive transforaminal lumbar interbody fusion (TLIF) compared with open procedures.

As with any operative technique, the operating surgeon's experience correlates with the efficacy and reliability of minimally invasive methods in the spine. ${ }^{11,27}$ However, the learning curve is steep with the use of MISS techniques..$^{26}$ To our knowledge there are no data in the literature regarding the impact of patient's body habitus and surgeon's learning experience on radiation emission during MISS. The aims of this study are 1) to analyze the amount of radiation emitted during various spine procedures performed with minimally invasive techniques; 2) to evaluate the impact of body habitus of patients on the fluoroscopic radiation emission during MISS; and 3) to demonstrate simultaneously if there is any association between the surgeon's expanding experience and radiation emission especially with regard to the obesity status of the patients.

\section{Methods \\ Study Design}

Institutional review board approval was obtained for this study. We performed a retrospective analysis of 332 patients who underwent 387 MISS procedures from January 2010 to August 2013. The majority of procedures were performed by the senior author (A.H.S.), who is a fellowship-trained spine surgeon and has been practicing spine surgery since late 2007. In a few cases, although the procedures were primarily performed by the senior author, residents and fellows were also involved at various steps. We accessed our institution's records, and patients who underwent MISS techniques were selected for inclusion. Although the senior author started his practice in late 2007, due to unavailability of the radiation emission dose for the older fluoroscopic equipment, analysis was performed using the information available from January 2010 onward. Age, sex, year of surgery, surgical procedure, levels of surgery, sides (unilateral/bilateral), radiation dose emitted, and BMI were recorded for each case. Microdiscectomy was performed in 131 patients, TLIF in 176 patients, and decompression with laminotomy in 80 patients. Since a fluoroscope was used only to determine the level of surgery in microdiscectomy and decompression procedures, these procedures were analyzed as a single group (microdiscectomy/decompression [MiDD], $\mathrm{n}$ $=211$ ). Patients who underwent TLIFs were divided into those undergoing unilateral TLIF (UnTLIF, $\mathrm{n}=106$ ) and bilateral TLIF (BiTLIF, $\mathrm{n}=70$ ). These 3 groups consti- tuted the major groups in this study. The OEC 9900 Elite (GE Healthcare), a uniplanar fluoroscopy device, was used during these procedures. The equipment was operated by certified technicians who varied throughout the study period. The fluoroscope was set in the automatic mode. The amount of radiation dose emitted is provided on this equipment. These levels were available in our institution's electronic records. In cases in which more than 1 level was treated, the radiation dose emitted was divided by the number of levels treated to estimate the dose for each level. Cases in which TLIF was performed at more than 1 level were not included because the involvement of an additional level does not correspond with the addition of equal numbers of pedicle screws. Patients who underwent 2 different procedures at 2 different levels were not included because of the unavailability of a separate radiation dose for each procedure. WHO criteria were used to define obesity, and the patients were divided into 6 categories: underweight (BMI < 18.50), normal (18.50-24.99), overweight (25.00-29.99), Class 1 obese (30.00-34.99), Class 2 obese (35.00-39.99), and Class 3 obese $(>40.00){ }^{31}$

\section{Surgical Technique}

The most common indication for surgery was herniated disc $(34.1 \%)$, followed by stenosis with degenerative disc disease (DDD) (28.3\%), stenosis alone (21.5\%), and spondylolisthesis (16.1\%). Standard MISS techniques were used. The MATRIX spine system (Synthes) was used in all cases. A 22-gauge spinal needle was used under C-arm fluoroscopy to find the most optimal spot on the skin to gain access to the operative level. A paramedian oblique approach was taken at $1.5 \mathrm{~cm}$ for decompression alone and $4.5 \mathrm{~cm}$ for TLIF from the midline. The length of the incision was $1.5 \mathrm{~cm}$ for MiDD and 1 inch for TLIF with the center of the incision at the initial finding needle. Insertion of the guidewire under image guidance was an initial and very important step. The guidewire was aimed directly toward the disc space at the desired level. The track over the guidewire was gradually dilated with the use of dilators of increasing size. A tubular retractor was inserted over the last dilator and was fixed to the table-mounted retractor holder. The typical diameter of the tubular retractor was $16 \mathrm{~mm}$ for MiDD and $22 \mathrm{~mm}$ for TLIF. Microsurgical methods were used to perform the desired procedure. In the TLIF procedures, guidewires were inserted in the pedicles for the screw placement prior to retractor placement. Microdiscectomy consisted of laminotomy, resection of the ligamentum flavum, foraminotomy, retraction of the nerve root, and excision of disc material. Important steps in TLIF procedures were resection of the ligamentum flavum, complete facetectomy, excision of disc material, insertion of an interbody cage filled with morcellized auto-/allograft, and instrumentation, either unilateral or bilateral (a majority of which was for spondylolisthesis corrections). In decompression alone, following the resection of the ligamentum flavum, laminotomy and foraminotomy were performed.

\section{Statistical Analysis}

Pearson's correlation test was used to demonstrate the 
linear relationship between radiation dose emission and BMI. Correlation coefficient is denoted as the $\mathrm{r}$ value. Median radiation dose emission in different groups was compared using the Kruskal-Wallis test. A post hoc test was used to perform a pairwise comparison within the group. Analyses were performed using the SPSS software (version 21 , IBM Inc.); $p<0.05$ was considered statistically significant.

\section{Results}

The mean age of the patients was 53.56 years (range 16-87 years) with almost equal numbers of male and female patients (164 and 168, respectively). A total of 387 MISS procedures were performed in 332 patients from January 2010 to August 2013 (Fig. 1). Sixty-five procedures were performed in 2010, 107 in 2011, 121 in 2012, and 94 in 2013 (until August). The majority of the surgeries were performed at 1 level $(93.7 \%)$. Overall, the radiation emission was highest during BiTLIF (median 113 mGy, SD 9.44 mGy, p < 0.001), whereas microdiscectomy required less exposure (12.62 mGy, SD $2.75 \mathrm{mGy}$; Fig. 2). Exposure time was also longer during BiTLIF compared with other procedures (mean exposure time 151.13 seconds for BiTLIF; 91.82 seconds for UnTLIF, MiDD = 20.67 seconds for MiDD).

\section{Body Habitus and Radiation Emission}

During MiDD, the radiation exposure was consistently higher for obese patients (Table 1). There was also a positive correlation between the radiation dose and the BMI of the patients $(\mathrm{r}=0.36, \mathrm{p}<0.001)$. The median radiation exposure was significantly higher for obese patients (Kruskal-Wallis test $p<0.001$ ). A substantial increase in radiation emission was observed in cases in which the patients'

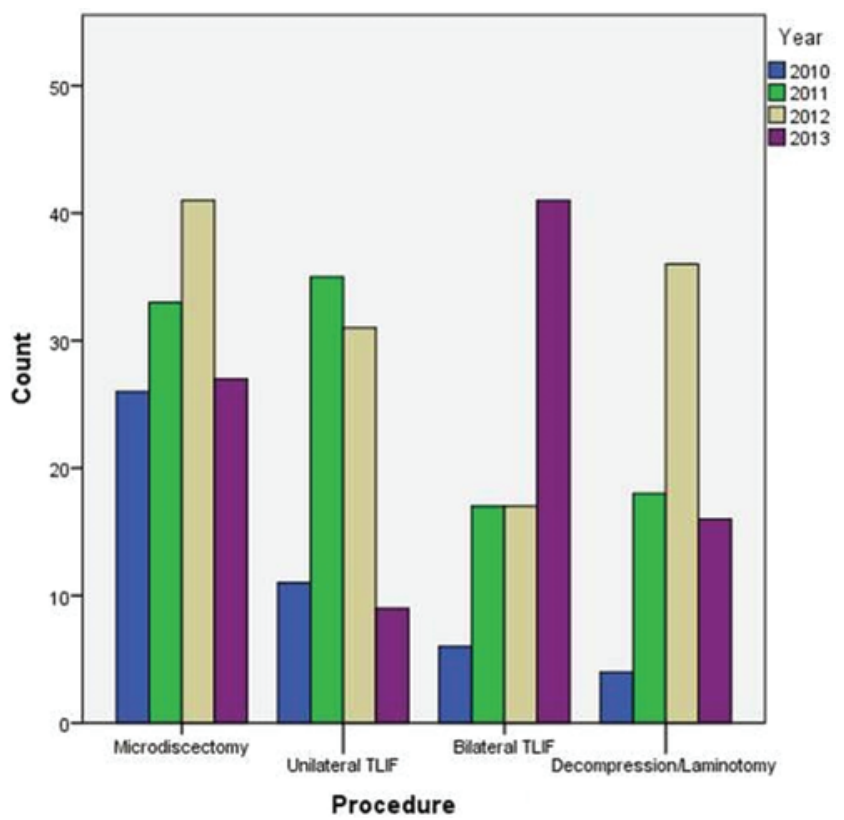

FIG. 1. Bar graph showing the number of minimally invasive spine procedures performed in the last 4 years. Figure is available in color online only.
BMI was greater than 30 (Fig. 3). On post hoc analysis, radiation exposure was significantly higher $(\mathrm{p}<0.001)$ for patients with Class 2 and Class 3 obesity (BMI $\geq 35)$ compared with other weight categories, although there was no difference between the Class 2 and Class 3 obesity groups $(\mathrm{p}=0.452)$. During TLIF, bilateral instrumentation contributed to more radiation emission than unilateral fixation (Table 1). Hence, a separate analysis was performed for these groups. There was a positive correlation between radiation emission during UnTLIF and patient BMI $(\mathrm{r}=$ $0.56, \mathrm{p}<0.001)$. The median dose of radiation was also higher in obese patients (Kruskal-Wallis test, $\mathrm{p}<0.001$ ). Radiation emission gradually increased in cases in which the patients' BMI was greater than 25 (overweight), although a more substantial increase was observed beyond Class 2 obesity (Fig. 4). A post hoc test also revealed a significant impact of Class 3 obesity $(\mathrm{p}<0.001)$ compared with other weight categories. Radiation exposure for patients with spondylolisthesis was not significantly different from that for patients with DDD+stenosis $(\mathrm{p}=0.287)$, although some quantitative difference was seen in very obese patients (Fig. 4).

Similarly, a positive correlation between radiation exposure and BMI was also observed in BiTLIF $(r=0.37$, $p$ $=0.002$ ). The median amount of radiation exposure was also significantly higher for obese patients (Kruskal-Wallis test, $\mathrm{p}=0.001$ ). Except for a decrease in the median value from Class 2 to Class 3 obesity, there was a gradual increase in radiation emission from lower to higher weight categories (Fig. 5). Significant influence of Class 2 obesity was also revealed on post hoc analysis $(\mathrm{p}=0.001)$, although there was no statistical difference in radiation exposure between the Class 2 and Class 3 obesity groups $(\mathrm{p}=0.542)$. Radiation exposure seems greater for patients undergoing BiTLIF for spondylolisthesis than for those undergoing the procedure for DDD+stenosis (Fig. 5), although the observation was not statistically significant ( $p$ $=0.148)$.

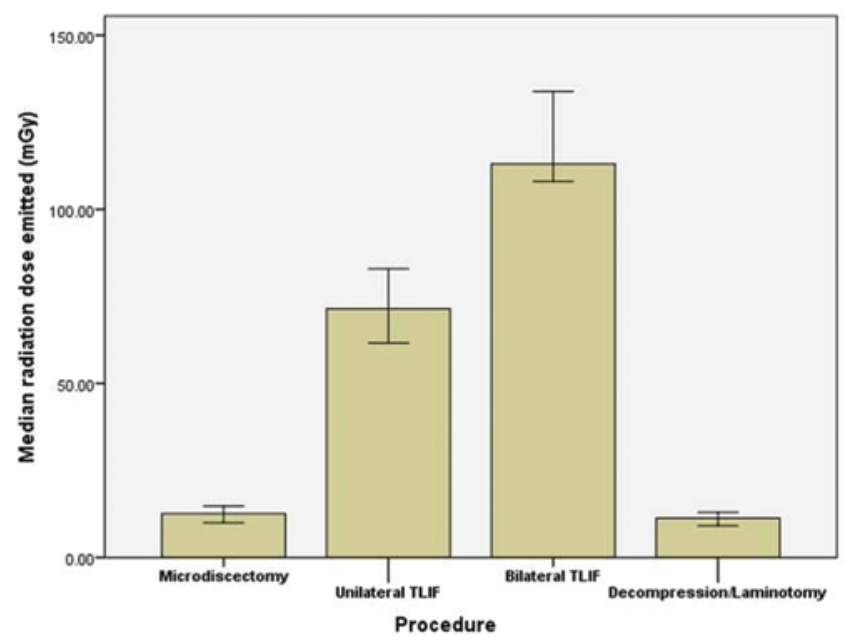

FIG. 2. Bar graph showing the median dose of radiation emission during various minimally invasive spine surgeries. Radiation exposure was significantly greater during BiTLIFs $(p<0.001)$. Error bars indicate SD. Figure is available in color online only. 
TABLE 1. Radiation emission during various MISS procedures

\begin{tabular}{|c|c|c|c|c|c|}
\hline \multirow{2}{*}{$\begin{array}{l}\text { Procedure \& Obesity Status } \\
\text { (WHO definition) }\end{array}$} & \multicolumn{5}{|c|}{ Median Radiation Emission (mGy) } \\
\hline & 2010 & 2011 & 2012 & 2013 & Overall \\
\hline \multicolumn{6}{|l|}{ MiDD } \\
\hline Underweight & - & - & - & - & - \\
\hline Normal & 12.53 & 4.58 & 8.78 & 9.58 & 6.77 \\
\hline Overweight & 13.42 & 8.19 & 11.86 & 9.24 & 9.89 \\
\hline Class 1 obesity & 19.30 & 9.96 & 12.50 & 12.70 & 10.99 \\
\hline Class 2 obesity & 32.42 & 25.87 & 24.31 & 21.52 & 23.47 \\
\hline Class 3 obesity & 65.17 & 37.64 & 31.17 & - & 35.36 \\
\hline \multicolumn{6}{|l|}{ UnTLIF } \\
\hline Underweight & - & - & - & - & - \\
\hline Normal & 58.95 & 57.23 & 42.80 & 61.46 & 55.84 \\
\hline Overweight & 64.24 & 60.89 & 54.90 & - & 55.30 \\
\hline Class 1 obesity & 100.02 & 67.69 & 65.31 & 66.96 & 67.42 \\
\hline Class 2 obesity & 103.45 & 112.82 & 89.09 & 84.56 & 88.79 \\
\hline Class 3 obesity & - & 232.56 & 285.98 & 123.67 & 154.26 \\
\hline \multicolumn{6}{|l|}{ BiTLIF } \\
\hline Underweight & 81.32 & - & - & - & 81.32 \\
\hline Normal & 165.89 & 152.91 & 70.62 & 72.41 & 85.15 \\
\hline Overweight & 188.06 & 113.00 & 95.95 & 97.53 & 106.04 \\
\hline Class 1 obesity & 224.80 & 148.90 & 119.21 & 123.78 & 133.90 \\
\hline Class 2 obesity & 254.56 & 299.48 & 156.55 & 128.75 & 199.00 \\
\hline Class 3 obesity & 286.00 & - & 120.34 & 213.44 & 168.00 \\
\hline
\end{tabular}

$-=$ There were no patients in these categories.

\section{Surgeon's Experience, Radiation Emission, and Obesity}

In most of the categories, the radiation emission was lower in procedures performed during 2013 than in 2010 (Table 1). There was a $23.5 \%$ reduction in radiation emission during MiDD from 2010 to 2013 in normal-weight patients. The reduction was observed to be more in the Class $1(34.2 \%)$ and Class 2 obesity (33.6\%) groups. Radiation emission had a slight increase (4.2\%) from 2010 to 2013 in normal-weight patients undergoing UnTLIF. Conversely, $33.1 \%$ and $18.3 \%$ reduction was seen in Class 1 and Class 2 obese patients, respectively. During BiTLIFs, the median radiation emission was approximately half in 2013 compared with 2010, which was seen in almost all weight categories. However, none of these observations were statistically significant $(\mathrm{p}>0.05)$.

\section{Discussion}

The term "minimally invasive surgery" was first coined by Wickham in $1987 .{ }^{30}$ Execution of operative procedures through a smaller incision and minimal soft-tissue dissection offers benefits to patients in terms of less operative pain and blood loss, and shorter hospital stay and recovery period. ${ }^{7,17}$ Fluoroscopic equipment is an important addition in the armamentarium of spine surgery to perform these techniques. However, the increasing popularity of MISS in recent years has also raised the concern of a higher risk of radiation exposure to all personnel in the operating room. ${ }^{1,6,12,19}$

There are 2 types of harmful effects of radiation expo- sure: acute, or deterministic, effects and late, or stochastic, effects. Acute or deterministic effects (erythema, epilation, and dermal necrosis) occur due to a high dose of radiation exposure, whereas stochastic effects (carcinogenesis and hereditary disorders) can occur at radiation doses lower than the dose limits of the various organs of our body.9,22 According to the National Council on Radiation Protection and Measurement, yearly occupational exposure limit for the body is 5 roentgen equivalent in man (rem), 50 rem for the extremity, and 15 rem for the eyes. ${ }^{16}$ Mariscalco et al. ${ }^{12}$ compared radiation exposure between open versus MISS microdiscectomy and observed significantly more radiation exposure in MISS than in open procedures. However, microdiscectomy performed using MISS techniques still appears to be safe, because the number of cases required to exceed the occupational exposure limit for the whole body and extremity is substantially large (1623 and 11,235 cases, respectively). Bindal et al. ${ }^{3}$ reported that the radiation exposures were within tolerable limits during minimally invasive TLIF. The baseline spontaneous cancer risk in the general US population is estimated to be $20 \%$, which shows an additional increase of $0.004 \%$ per $\mathrm{mSv}$ of radiation exposure.,16 The cumulative risk may be substantial over several years of a surgeon's operative practice. ${ }^{12}$ Owing to substantial radiation exposure to all operating room personnel, several guidelines and recommendations are outlined to limit the radiation dose (Table 2). ${ }^{1-3,10,12,18}$ There is a paucity of literature describing a quantitative association of patient size with the radiation exposure during spine surgeries, including minimally 

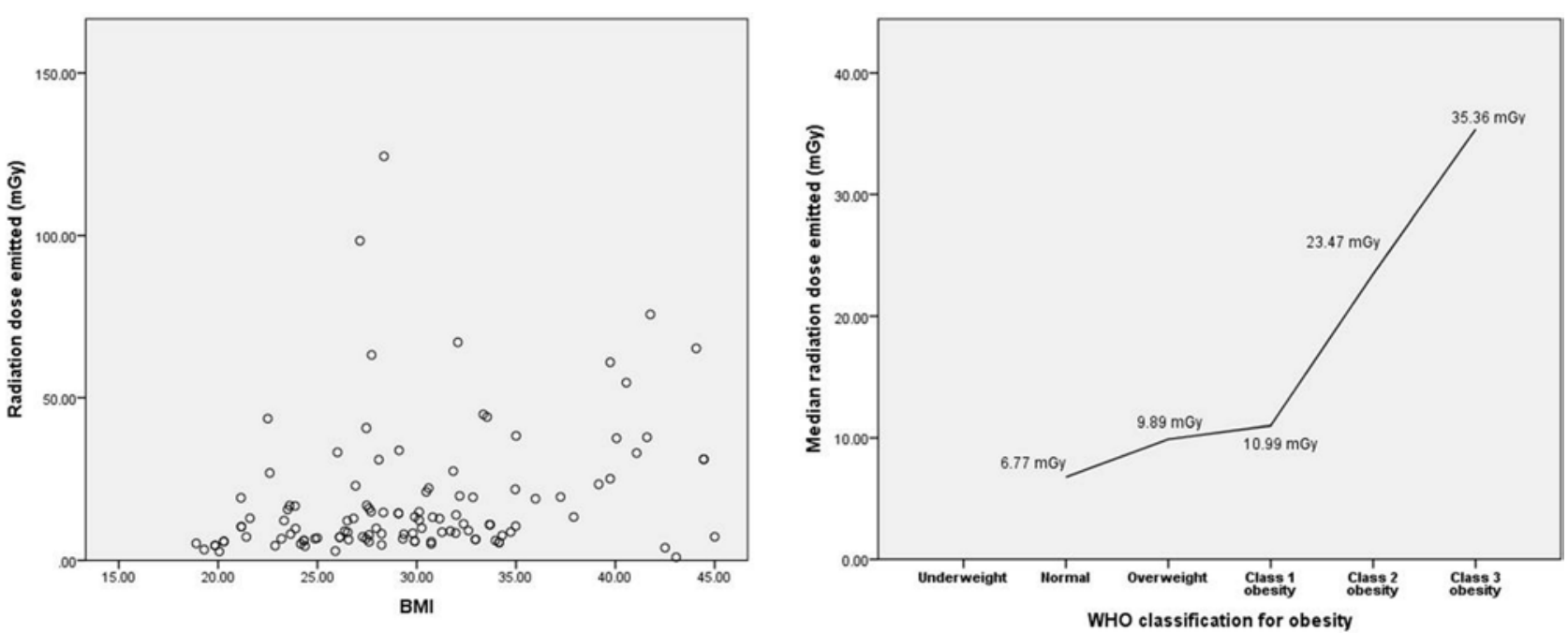

FIG. 3. Radiation exposure during MiDD. Left: Positive correlation was seen between radiation emission and BMI $(r=0.36, p<$ 0.001). Right: Median radiation emission was significantly higher in cases involving obese patients (Kruskal-Wallis test $p<0.001)$. A substantial increase in radiation emission was observed beyond a BMI of 30 .

invasive procedures. In the present study, we analyzed the effect of patients' body habitus on radiation emission during MISS and also identified the critical BMI levels, which led to a significant increase in radiation emission during various procedures.

A varying amount of radiation exposure among different patients and procedures can be explained by fluoroscope-related technical factors. The quantity of the electron flow is described in units of milliamperes and the maximum kinetic energy of the accelerated electrons is defined as kilovolts peak. A higher milliampere value indicates more x-ray production, whereas increasing kilovolts peak of the equipment will result in production of more penetrating radiation. Although using the higher kilovolts peak reduces radiation exposure to the patient and other operating room personnel, it also reduces the relative image contrast between tissues of different densities. Setting the machine either under automatic or manual mode can substantially influence the amount of radiation emission. When the manual mode is used, the radiation exposure rate is independent of the patient's size; however, brightness of the image is adversely affected. For this reason, the fluoroscope is generally used in automatic brightness control. Whenever less radiation reaches the detector, to produce a sufficiently bright image the device adjusts its milliampere value, kilovolts peak, or both, which either increases the radiation exposure or increases the penetrating radiation or both, respectively, which also explains the greater radiation emission in cases in which obese patients are treated. ${ }^{3}$ At a fixed kilovolts peak the amount of radiation emission is the product of milliamperes and exposure time; hence, the emission is usually greater during the procedure requiring longer fluoroscopic exposure. We also observed greater radiation exposure during BiTLIF, which required longer fluoroscopic exposure (151.13 seconds) compared with other groups.
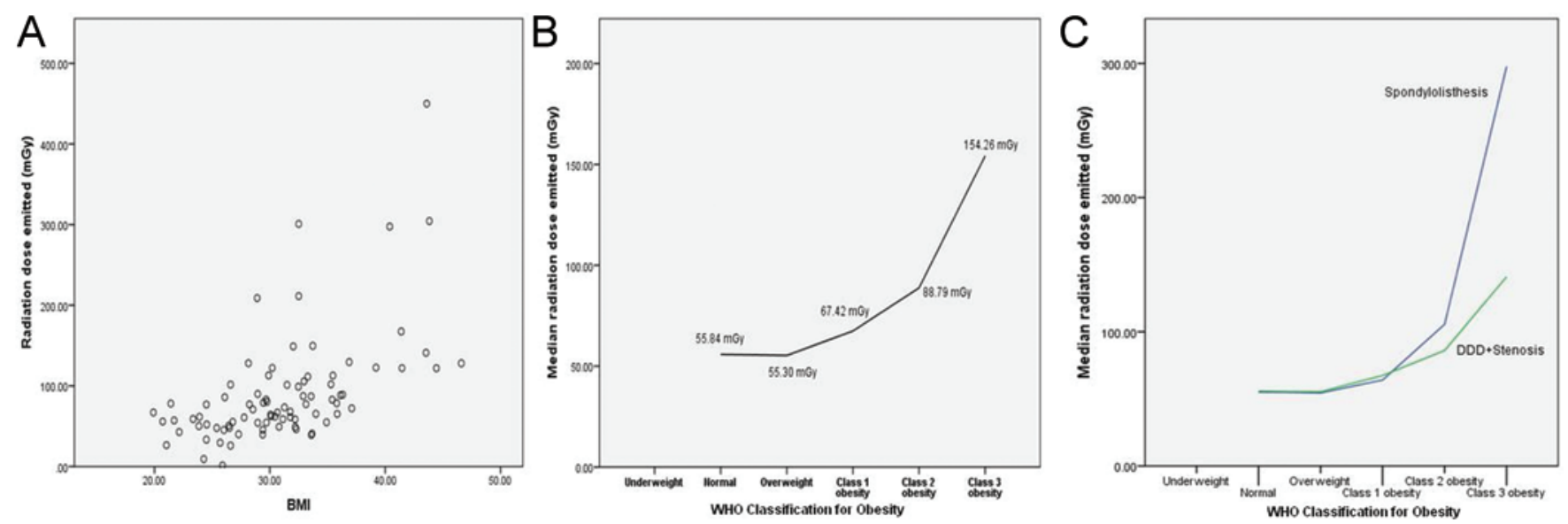

FIG. 4. Radiation exposure during UnTLIF. A: Positive correlation was seen between radiation emission and BMI $(r=0.56, p<$ 0.001). B: Radiation emission gradually increased beyond a BMI of 25 (overweight), although a more substantial increase was observed beyond Class 2 obesity $(\mathrm{BMI} \geq 35)$. C: Radiation emission in spondylolisthesis was not significantly different from patients having $\mathrm{DDD}+$ stenosis $(p=0.287)$, although some quantitative difference was seen in very obese patients. Figure is available in color online only. 

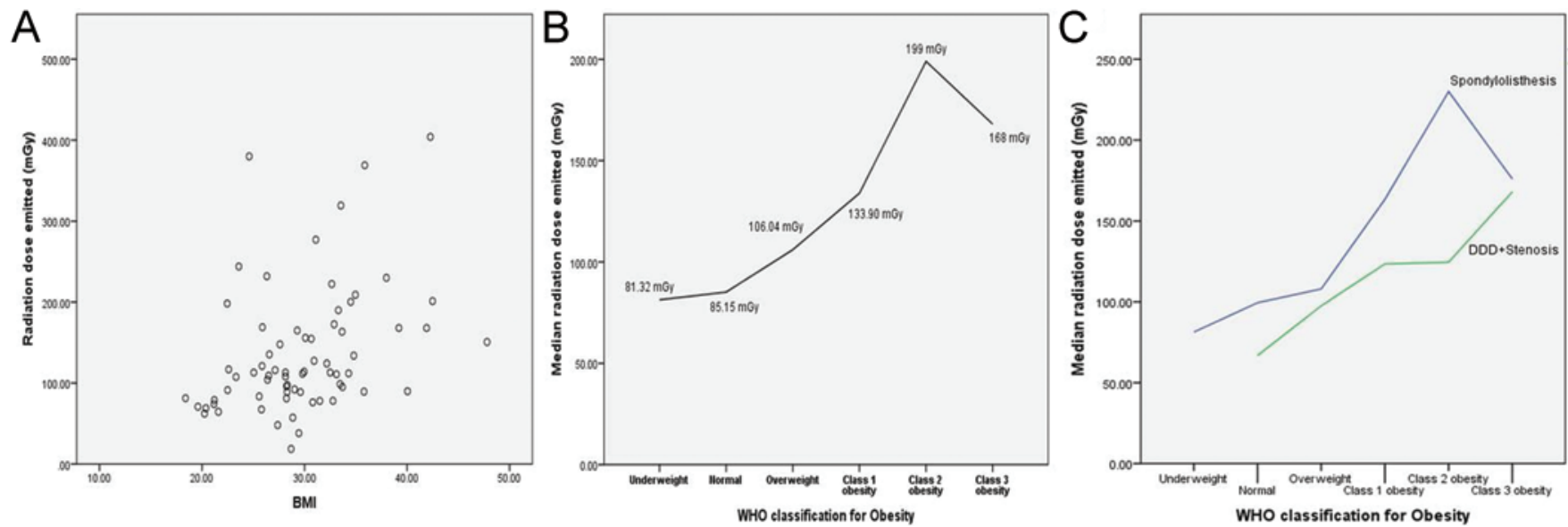

FIG. 5. Radiation exposure during BiTLIF. A: Positive correlation was seen between radiation emission and BMI $(r=0.36, p=$ 0.002). B: Except for a decrease in the median value from Class 2 to Class 3 obesity, there was a gradual increase in radiation emission from lower to higher weight categories. C: Radiation emissions seem greater during BiTLIF for spondylolisthesis than for $\mathrm{DDD}+$ stenosis, although the observation was not statistically significant $(p=0.148)$. Figure is available in color online only.

We consistently observed a statistically significant positive correlation between the amount of radiation emission and BMI of the patients during all types of MISS procedures. The median dose of radiation emission was also significantly higher in patients with a large body habitus in all treatment groups. When performing the post hoc analysis, we observed that the radiation emission during MiDD and BiTLIF was significantly higher after a BMI cutoff level of 35 (Class 2 and 3 obesity). On the other hand, this cutoff level was higher ( $\geq 40$, Class 3 obesity) during UnTLIF. Apart from more radiation absorption in larger patients, intraoperative technical challenges are also responsible for more radiation emission in obese patients. Greater skin-tofascia and fascia-to-spine depth obstructs the maneuvering of the smaller MISS instruments, which is also responsible for further increase in the operative time and radiation exposure. Having considered these findings, it seems imperative that the advantages of MISS should be weighed against the amount of radiation exposure during such pro- cedures in markedly obese patients. However, Wang et al. ${ }^{28}$ reported several benefits in the immediate postoperative period of performing minimally invasive TLIFs in overweight and obese patients, but long-term outcomes including the fusion rates were the same as with the open technique.

MISS is also a technically demanding procedure. 3D anatomical orientation is reduced, which makes it difficult to manage intraoperative complications. ${ }^{21}$ The technical dependency associated with MISS accounts for a steep, long, and often a complication-ridden learning curve. ${ }^{11,21}$ A surgeon's experience has significant impact on the effectiveness and safety of the MISS procedures. ${ }^{11,27}$ In our study, the senior author (A.H.S.) performed more procedures during 2013 (94 procedures by August) than in 2010. We observed that generally the amount of radiation emission was less in 2013 than that in 2010 (except slightly more in UnTLIFs in normal-weight patients, Table 1). We also noted that, from 2010 to 2013, this reduction in radia-

TABLE 2. Steps to minimize radiation exposure to all operating team personnel

\begin{aligned} \hline Step & Description \\ \hline 1 & Use of protective devices (thyroid shields, leaded glasses, lead aprons, \& lead gloves) \\ \hline 2 & Use of "hand-off" technique \\ \hline 3 & Annual radiation exposure monitoring of operating staff with dosimeters \\ \hline 5 & Personnel should stand opposite the x-ray source \\ \hline 6 & Inferior position of the x-ray tube \\ \hline 8 & $\begin{array}{c}\text { Taking intermittent images on fluoroscope } \\ \text { Use "time-distance-shielding" principle: minimizing duration of exposure; maintaining a sufficient distance from the } \\ \text { source; \& use shielding }\end{array} \\$\hline 10 & $\begin{array}{c}\text { In the lateral view, the source should be positioned with the radiation beam going away from the operator } \\ \text { pedicle access needle \& tap, which allows limited number of fluoroscopic images }\end{array} \\$\hline 11 & $\begin{array}{c}\text { Operating the C-arm by surgeon using the footswitch } \\ \text { Educating the operating team personnel about the radiation hazards \& display of directives on minimizing the radia- } \\ \text { tion exposure in the operating room }\end{array} \\$\hline & $\quad\end{aligned}$


tion exposure was greater in large patients. However, the aforementioned observations regarding the effect of the surgeon's learning curve were not statistically relevant.

\section{Strengths and Limitations of Our Study}

Our study is the first of its kind to describe the effect of the patient's BMI status on the amount of radiation emission during MISS. Effectiveness of MISS with the growing experience of the operating surgeon has been commonly discussed. ${ }^{11,26,27}$ A goal of this study was to identify the impact of the surgeon's learning curve on fluoroscopic radiation emission with specific consideration of the patient's body habitus. However, there are some limitations to our study, which restrict the general implication of the findings. Involvement of residents and fellows in a few cases may affect the amount of radiation emission. As technicians varied during the study period, the involvement of different technicians from case to case could also influence the results. We did not evaluate the clinical and radiological results; thus, any association of radiation emission with the functional outcomes and accuracy of hardware placement could not be demonstrated. Because of unavailability of fluoroscopic radiation data during the early years of the senior author's clinical practice, any interpretation of the impact of the surgeon's learning experience should be carefully made. We also believe that for this reason, we did not observe a significant impact of the surgeon's experience on the radiation emission. The surgeon was likely already in a later phase on the learning curve at the beginning of the study in 2010. Another limitation of our study is that we did not use dosimeters to estimate the radiation exposure to the specific organs of the operating team personnel.

\section{Conclusions}

We present the first study describing the impact of body habitus on radiation emission in MISS techniques. Fluoroscopic radiation emission is significantly higher during MISS procedures performed in larger patients. Severe obesity ( $\mathrm{BMI} \geq 35)$ is associated with a significantly greater risk of radiation exposure compared with other weight categories. We do believe that a surgeon's operative experience is one of the important factors that contributes to limiting exposure to radiation during MISS especially in obese patients. Unavailability of radiation emission doses during the early phase of the senior author's surgical practice is a major limitation to our study. However, this analysis can be an important guide to future studies to demonstrate this association.

\section{References}

1. Ahn Y, Kim CH, Lee JH, Lee SH, Kim JS: Radiation exposure to the surgeon during percutaneous endoscopic lumbar discectomy: a prospective study. Spine (Phila Pa 1976) 38:617-625, 2013

2. Bar-On E, Weigl DM, Becker T, Katz K, Konen O: Intraoperative $\mathrm{C}$-arm radiation affecting factors and reduction by an intervention program. J Pediatr Orthop 30:320-323, 2010

3. Bindal RK, Glaze S, Ognoskie M, Tunner V, Malone R, Ghosh S: Surgeon and patient radiation exposure in minimally invasive transforaminal lumbar interbody fusion. J Neurosurg Spine 9:570-573, 2008
4. Boring CC, Squires TS, Tong T: Cancer statistics, 1991. CA Cancer J Clin 41:19-36, 1991

5. Elgafy H, O'Brien P, Blessinger B, Hassan A: Challenges of spine surgery in obese patients. Am J Orthop 41:E46-E50, 2012

6. Fransen P: Fluoroscopic exposure in modern spinal surgery. Acta Orthop Belg 77:386-389, 2011

7. Harrington JF, French P: Open versus minimally invasive lumbar microdiscectomy: comparison of operative times, length of hospital stay, narcotic use and complications. Minim Invasive Neurosurg 51:30-35, 2008

8. Hsi RS, Zamora DA, Kanal KM, Harper JD: Severe obesity is associated with 3 -fold higher radiation dose rate during ureteroscopy. Urology 82:780-785, 2013

9. International Commission on Radiological Protection: A report on progress towards new recommendations: a communication from the International Commission on Radiological Protection. J Radiol Prot 21:113-123, 2001

10. Jones DP, Robertson PA, Lunt B, Jackson SA: Radiation exposure during fluoroscopically assisted pedicle screw insertion in the lumbar spine. Spine (Phila Pa 1976) 25:15381541,2000

11. Lee JC, Jang HD, Shin BJ: Learning curve and clinical outcomes of minimally invasive transforaminal lumbar interbody fusion: our experience in 86 consecutive cases. Spine (Phila Pa 1976) 37:1548-1557, 2012

12. Mariscalco MW, Yamashita T, Steinmetz MP, Krishnaney AA, Lieberman IH, Mroz TE: Radiation exposure to the surgeon during open lumbar microdiscectomy and minimally invasive microdiscectomy: a prospective, controlled trial. Spine (Phila Pa 1976) 36:255-260, 2011

13. Mayer HM, Brock M: Percutaneous endoscopic discectomy: surgical technique and preliminary results compared to microsurgical discectomy. J Neurosurg 78:216-225, 1993

14. Mroz TE, Abdullah KG, Steinmetz MP, Klineberg EO, Lieberman IH: Radiation exposure to the surgeon during percutaneous pedicle screw placement. J Spinal Disord Tech 24:264-267, 2011

15. Mroz TE, Yamashita T, Davros WJ, Lieberman IH: Radiation exposure to the surgeon and the patient during kyphoplasty. J Spinal Disord Tech 21:96-100, 2008

16. National Council on Radiation Protection and Measurements: Recommendations on Limits for Exposure to Ionizing Radiation. Report No. 91. Bethesda, MD: NCRP, 1987

17. Oppenheimer JH, DeCastro I, McDonnell DE: Minimally invasive spine technology and minimally invasive spine surgery: a historical review. Neurosurg Focus 27(3):E9, 2009

18. Ortiz AO, Natarajan V, Gregorius DR, Pollack S: Significantly reduced radiation exposure to operators during kyphoplasty and vertebroplasty procedures: methods and techniques. AJNR Am J Neuroradiol 27:989-994, 2006

19. Payer M: "Minimally invasive" lumbar spine surgery: a critical review. Acta Neurochir (Wien) 153:1455-1459, 2011

20. Peng CW, Bendo JA, Goldstein JA, Nalbandian MM: Perioperative outcomes of anterior lumbar surgery in obese versus non-obese patients. Spine J 9:715-720, 2009

21. Perez-Cruet MJ, Fessler RG, Perin NI: Review: complications of minimally invasive spinal surgery. Neurosurgery 51 (5 Suppl):S26-S36, 2002

22. Perisinakis K, Theocharopoulos N, Damilakis J, Katonis P, Papadokostakis G, Hadjipavlou A, et al: Estimation of patient dose and associated radiogenic risks from fluoroscopically guided pedicle screw insertion. Spine (Phila Pa 1976) 29: 1555-1560, 2004

23. Rampersaud YR, Foley KT, Shen AC, Williams S, Solomito M: Radiation exposure to the spine surgeon during fluoroscopically assisted pedicle screw insertion. Spine (Phila Pa 1976) 25:2637-2645, 2000

24. Synowitz M, Kiwit J: Surgeon's radiation exposure during 
percutaneous vertebroplasty. J Neurosurg Spine 4:106-109, 2006

25. Tsivian M, Abern MR, Yoo JJ, Evans P, Qi P, Kim CY, et al: Radiation exposure associated with dedicated renal mass computed tomography protocol: impact of patient characteristics. J Endourol 27:1102-1106, 2013

26. Wang B, Lü G, Patel AA, Ren P, Cheng I: An evaluation of the learning curve for a complex surgical technique: the full endoscopic interlaminar approach for lumbar disc herniations. Spine J 11:122-130, 2011

27. Wang H, Huang B, Li C, Zhang Z, Wang J, Zheng W, et al: Learning curve for percutaneous endoscopic lumbar discectomy depending on the surgeon's training level of minimally invasive spine surgery. Clin Neurol Neurosurg 115:19871991,2013

28. Wang J, Zhou Y, Feng Zhang Z, Qing Li C, Jie Zheng W, Liu $\mathrm{J}$ : Comparison of clinical outcome in overweight or obese patients after minimally invasive versus open transforaminal lumbar interbody fusion. J Spinal Disord Tech 27:202-206, 2014

29. Weiss DJ, Pipinos II, Longo GM, Lynch TG, Rutar FJ, Johanning JM: Direct and indirect measurement of patient radiation exposure during endovascular aortic aneurysm repair. Ann Vasc Surg 22:723-729, 2008
30. Wickham JE: The new surgery. Br Med J (Clin Res Ed) 295:1581-1582, 1987

31. World Health Organization: Obesity: Preventing and Managing the Global Epidemic. Geneva: World Health Organization, 2000

\section{Author Contributions}

Conception and design: Sin, Kukreja. Acquisition of data: Kukreja, Haydel. Analysis and interpretation of data: Kukreja, Haydel. Drafting the article: Kukreja, Haydel. Critically revising the article: Sin, Nanda. Reviewed submitted version of manuscript: Sin, Nanda. Approved the final version of the manuscript on behalf of all authors: Sin. Statistical analysis: Kukreja, Haydel. Administrative/technical/material support: Sin, Nanda. Study supervision: $\operatorname{Sin}$.

\section{Correspondence}

Anthony H. Sin, Department of Neurosurgery, Louisiana State University Health Science Center-Shreveport, P.O. Box 33932, 1501 Kings Highway, Shreveport, LA 71130-3932. email: asin@ lsuhsc.edu. 